\title{
Note on one inequality and its application in intuitionistic fuzzy sets theory. Part 1
}

\author{
Mladen V. Vassilev-Missana \\ 5 Victor Hugo Str., Sofia, Bulgaria \\ e-mail: missana@abv.bg
}

Received: 20 October 2020

Accepted: 7 March 2021

\begin{abstract}
The inequality $\mu^{\frac{1}{\nu}}+\nu^{\frac{1}{\mu}} \leq \frac{1}{2}$ is introduced and proved, where $\mu$ and $\nu$ are real numbers, for which $\mu, \nu \in[0,1]$ and $\mu+\nu \leq 1$. The same inequality is valid for $\mu=\mu_{A}(x)$, $\nu=\nu_{A}(x)$, where $\mu_{A}$ and $\nu_{A}$ are the membership and the non-membership functions of an arbitrary intuitionistic fuzzy set $A$ over a fixed universe $E$ and $x \in E$. Also, a generalization of the above inequality for arbitrary $n \geq 2$ is proposed and proved.
\end{abstract}

Keywords: Inequality, Intuitionistic fuzzy sets.

2020 Mathematics Subject Classification: 03 E72.

\section{Introduction}

The Intuitionistic Fuzzy Sets (IFSs) are introduced by K. Atanassov in [1,2] as follows. Let $E$ be a universal set, $\mu_{A}, \nu_{A}: E \rightarrow I:=[0,1]$ be mappings and for each $x \in E$ :

$$
\mu_{A}(x)+\nu_{A}(x) \leq 1 .
$$

Then the set

$$
A=\left\{\left\langle x, \mu_{A}(x), \nu_{A}(x)\right\rangle \mid x \in E\right\}
$$

is called an IFS.

Mappings $\mu_{A}$ and $\nu_{A}$ are called membership and non-membership functions for the element $x \in E$ to the set $A \subseteq E$.

When for each $x \in E$ :

$$
\mu_{A}(x)+\nu_{A}(x)=1,
$$

the set $A$ is transformed to the ordinary fuzzy (Zadeh's) set [4]. 


\section{Main results}

The main result of the paper is the following Theorem 1 .

Theorem 1. Let $\mu, \nu \in I$ be real numbers satisfying inequality

$$
\mu+\nu \leq 1
$$

Then the inequality

$$
\mu^{\frac{1}{\nu}}+\nu^{\frac{1}{\mu}} \leq \frac{1}{2}
$$

holds, where the equality is possible if and only if $\mu=\nu=\frac{1}{2}$.

Before giving the proof of Theorem 1, we need the following lemma.

Lemma. Let the function $f$ be given by

$$
f(x)=(1-x)^{\frac{1}{x}}:=e^{\frac{\ln (1-x)}{x}} .
$$

Then function $f$ is strictly concave on interval $(0,1)$ and also strictly decreasing on the same interval. Also, $f(1)=0$ and if we define $f(0):=\lim _{x \rightarrow 0^{+}} f(x)$, then $f(0)=\frac{1}{e}$.

Proof. Using (5) we obtain:

$$
\left(\frac{d}{d x}\right) f(x)=f(x) \cdot \frac{d}{d x}\left(\frac{\ln (1-x)}{x}\right)
$$

and

$$
\left(\frac{d}{d x}\right)^{2} f(x)=f(x) \cdot\left(\frac{d}{d x}\left(\frac{\ln (1-x)}{x}\right)\right)^{2}+\left(\frac{d}{d x}\right)^{2}\left(\frac{\ln (1-x)}{x}\right) .
$$

For $x \in(0,1)$ we have

$$
\ln (1-x)=-\sum_{n=0}^{\infty} \frac{x^{n+1}}{n+1}
$$

Hence,

$$
\frac{\ln (1-x)}{x}=-\sum_{n=0}^{\infty} \frac{x^{n}}{n+1}
$$

The series $\sum_{n=0}^{\infty} \frac{x^{n}}{n+1}$ converges uniformly on each compact set $\left[\delta_{1}, \delta_{2}\right]$, where $0<\delta_{1}<\delta_{2}<1$. This follows from Weierstrass criterion for uniform convergence of series (see [3]), since

$$
\sum_{n=0}^{\infty} \frac{x^{n}}{n+1} \leq \sum_{n=0}^{\infty} \frac{\delta_{2}^{n}}{n+1}
$$

and the series $\sum_{n=0}^{\infty} \frac{\delta_{2}^{n}}{n+1}$ converges (from D'Alembert criterion for convergence of series (see [3]). Therefore, (8) yields

$$
\frac{d}{d x}\left(\frac{\ln (1-x)}{x}\right)=-\sum_{n=0}^{\infty} \frac{(n+1)}{n+2} x^{n} .
$$


Since, $f(x)>0$, formulas (6) and (9) imply that $f$ is strictly decreasing on $(0,1)$.

The series $(9)$ converges uniformly on each compact set $\left[\delta_{1}, \delta_{2}\right]$, where $0<\delta_{1}<\delta_{2}<1$. Therefore,

$$
\left(\frac{d}{d x}\right)^{2}\left(\frac{\ln (1-x)}{x}\right)=-\sum_{n=0}^{\infty} \frac{(n+1)(n+2)}{n+3} x^{n}
$$

and the series (10) converges (from D'Alembert criterion). Then, to prove that $f$ is strictly concave, we need to prove that

$$
\left(\frac{d}{d x}\right)^{2} f(x)<0
$$

for $x \in(0,1)$. Because of $(7),(9)$ and (10), inequality (11) is equivalent to the inequality

$$
\left(\sum_{n=0}^{\infty} \frac{(n+1)}{n+2} x^{n}\right)^{2}<\sum_{n=0}^{\infty} \frac{(n+1)(n+2)}{n+3} x^{n} .
$$

The left-hand side of inequality (12) must be understood as a Cauchy-Mertens multiplication of the series $\sum_{n=0}^{\infty} \frac{(n+1)}{n+2} x^{n}$ by itself, i.e.,

$$
\begin{aligned}
\left(\sum_{n=0}^{\infty} \frac{(n+1)}{n+2} x^{n}\right)^{2} & =\left(\sum_{n=0}^{\infty} \frac{(n+1)}{n+2} x^{n}\right) \cdot\left(\sum_{n=0}^{\infty} \frac{(n+1)}{n+2} x^{n}\right) \\
& =\sum_{n=0}^{\infty}\left(\sum_{k=0}^{n} \frac{k+1}{k+2} \cdot \frac{n-k+1}{n-k+2}\right) x^{n} .
\end{aligned}
$$

From (13), inequality (12) is equivalent to

$$
\sum_{n=0}^{\infty}\left(\sum_{k=0}^{n} \frac{k+1}{k+2} \cdot \frac{n-k+1}{n-k+2}\right) x^{n}<\sum_{n=0}^{\infty} \frac{(n+1)(n+2)}{n+3} x^{n} .
$$

To prove (14), it is enough to prove that the inequality

$$
\sum_{k=0}^{n} \frac{k+1}{k+2} \cdot \frac{n-k+1}{n-k+2}<\frac{(n+1)(n+2)}{n+3}
$$

holds. For this aim, we use the equality

$$
\frac{1}{n+4} \cdot\left(\frac{1}{k+2}+\frac{1}{n-k+2}\right)=\frac{1}{(k+2)(n-k+2)} .
$$

Because of (16), we may rewrite (15) in the form

$$
\sum_{k=0}^{n} \frac{1}{n+4} \cdot\left(\frac{1}{k+2}+\frac{1}{n-k+2}\right) \cdot(k+1)(n-k+1)<\frac{(n+1)(n+2)}{n+3} .
$$

But (17) is equivalent to

$$
\sum_{k=0}^{n} \frac{1}{n+4} \cdot \frac{k+1}{k+2} \cdot(n-k+1)+\sum_{k=0}^{n} \frac{1}{n+4} \cdot(k+1) \cdot \frac{n-k+1}{n-k+2}<\frac{(n+1)(n+2)}{n+3} .
$$


Since $\frac{k+1}{k+2}<1$ and $\frac{n-k+1}{n-k+2}<1,(18)$ will be proved if we know that the inequality

$$
\frac{1}{n+4} \cdot\left(\sum_{k=0}^{n}(n-k+1)+\sum_{k=0}^{n}(k+1)\right)<\frac{(n+1)(n+2)}{n+3} \text {. }
$$

holds. But after a simple calculation, the left-hand side of (19) equals to $\frac{(n+1)(n+2)}{n+4}$.

This proves (19) since

$$
\frac{(n+1)(n+2)}{n+4}<\frac{(n+1)(n+2)}{n+3} .
$$

Then the above mentioned function $f$ is strictly concave on interval $(0,1)$. Also,

$$
\lim _{x \rightarrow o^{+}} f(x)=\lim _{x \rightarrow o^{+}}(1-x)^{\frac{1}{x}}=\lim _{y \rightarrow \infty}\left(1-\frac{1}{y}\right)^{y}=\frac{1}{e}
$$

and the Lemma is proved.

Corollary 1. If $x \in(0,1)$ and $x \neq \frac{1}{2}$, then

$$
f(x)+f(1-x)<2 f\left(\frac{1}{2}\right)=2 \cdot \frac{1}{4}=\frac{1}{2} .
$$

When $x=\frac{1}{2},(20)$ is an equality.

Proof. Since $f$ is strictly concave on $(0,1)$, then

$$
\frac{f(x)+f(1-x)}{2}<f\left(\frac{x+(1-x)}{2}\right)=f\left(\frac{1}{2}\right)
$$

and (20) holds.

Corollary 2. If $\mu, \nu \in(0,1)$ and $\mu+\nu=1$, then (4) holds.

Proof. If $\mu=\nu=\frac{1}{2}$, then, obviously, (4) is an equality.

Let $\mu \neq \nu$. We put $\mu=1-x, \nu=x$. Hence, $x \in(0,1)$. Since

$$
f(x)+f(1-x)=(1-x)^{\frac{1}{x}}+x^{\frac{1}{1-x}},
$$

then (20) yields exactly (4).

We must note that Corollary 2 means that for fuzzy sets (4) is always true for $\mu, \nu \in I$, since

$$
f(0)=\frac{1}{e}<\frac{1}{2}
$$

and $f(1)=0$.

Proof of Theorem 1. From Corollary 1, we have that Theorem 1 is valid for $\mu+\nu=1$.

Let $\mu, \nu \in(0,1)$ and $\mu+\nu<1$. Also, let $\alpha=1-\mu$. Therefore, $\alpha \in(0,1)$. Then we have

$$
\mu^{\frac{1}{\alpha}}+\alpha^{\frac{1}{\mu}} \leq \frac{1}{2}
$$


But, also, we have

$$
0<\nu<\alpha<1
$$

and

$$
1<\frac{1}{\alpha}<\frac{1}{\nu}
$$

From $0<\mu<1$ and (23) we obtain

$$
\mu^{\frac{1}{\nu}}<\mu^{\frac{1}{\alpha}}
$$

From (22) we obtain

$$
\nu^{\frac{1}{\mu}}<\alpha^{\frac{1}{\mu}}
$$

Now, (24) and (25) yield

$$
\mu^{\frac{1}{\nu}}+\nu^{\frac{1}{\mu}}<\mu^{\frac{1}{\alpha}}+\alpha^{\frac{1}{\mu}} .
$$

From (21) and (26) inequality (4) holds immediately. Thus, Theorem 1 holds for the case $\mu, \nu \in(0,1)$.

It remains only to consider the following cases:

1. $\mu=0$,

2. $\mu=1$.

If Case 1 holds, we consider the subcases:

1.1. If $\nu=0$, then $\mu^{\frac{1}{\nu}}$ and $\nu^{\frac{1}{\mu}}$ takes the form $0^{\frac{1}{0}}$, which we may consider (after putting $\mu=x$ ) as

$$
\lim _{x \rightarrow 0^{+}} x^{\frac{1}{x}}=\lim _{y \rightarrow \infty}\left(\frac{1}{y}\right)^{y}=0 .
$$

Therefore, (4) is obviously true.

1.2. If $\nu \neq 0$ is true, then $\mu^{\frac{1}{\nu}}=0^{\frac{1}{\nu}}=0$.

Also, when $0<\nu<1, \nu^{\frac{1}{\mu}}=\nu^{+\infty}=0$.

If $\nu=1$, then $\nu^{\frac{1}{\mu}}$ takes the form $1^{+\infty}$, which we understand (after putting $\mu=x$ ) as

$$
\lim _{x \rightarrow 0^{+}}(1-x)^{\frac{1}{x}}=\lim _{y \rightarrow \infty}\left(1-\frac{1}{y}\right)^{y}=\frac{1}{e} .
$$

Therefore, (4) is true, since $\frac{1}{e}<\frac{1}{2}$.

Let Case 2 hold. Then $\nu=0$, because of the conditions $0 \leq \nu$ and $\mu+\nu=1$. Therefore,

$$
\nu^{\frac{1}{\mu}}=0^{1}=0 .
$$

Also, we have that $\mu^{\frac{1}{\nu}}$ takes the form $1^{+\infty}$, which (after putting $\nu=x$ ) we consider as

$$
\lim _{x \rightarrow 0^{+}}(1-x)^{\frac{1}{x}}=\lim _{y \rightarrow \infty}\left(1-\frac{1}{y}\right)^{y}=\frac{1}{e} .
$$

Therefore, (4) is again true and Theorem 1 is proved. 
Finally, we will give an unexpected form of Theorem 1 for the case of fuzzy sets.

Theorem 2. Let $\mu, \nu \in(0,1)$ and $\mu+\nu=1$. Then the inequality

$$
\mu^{1+\mu+\mu^{2}+\cdots}+\nu^{1+\nu+\nu^{2}+\cdots} \leq \frac{1}{2}
$$

holds and the equality is possible if and only if $\mu=\nu=\frac{1}{2}$.

Proof. We use (4) that is proved in Theorem 1. But since $\mu+\nu=1$, we may rewrite (4) in the form

$$
\mu^{\frac{1}{1-\mu}}+\nu^{\frac{1}{1-\nu}} \leq \frac{1}{2}
$$

Since $\mu, \nu \in(0,1)$, we have

$$
\frac{1}{1-\mu}=1+\mu+\mu^{2}+\ldots, \frac{1}{1-\nu}=1+\nu+\nu^{2}+\ldots
$$

Then, from (28) and (29) inequality (27) holds.

Let us now look at one generalization of (4) for arbitrary $n \geq 2$, proving the following theorem.

Theorem 3. Let $n \geq 2$ be an arbitrary integer and $x_{i} \in(0,1), i=1,2, \ldots, n$ be real numbers, such that

$$
\sum_{i=1}^{n} x_{i}=1
$$

Then the inequality

$$
\frac{\left(1-x_{1}\right)^{\frac{1}{x_{1}}}+\left(1-x_{2}\right)^{\frac{1}{x_{2}}}+\cdots+\left(1-x_{n}\right)^{\frac{1}{x_{n}}}}{n} \leq\left(1-\frac{1}{n}\right)^{n}
$$

holds and the equality is possible if and only if $x_{1}=x_{2}=\cdots=\frac{1}{n}$.

Proof. Since $f(x)=(1-x)^{\frac{1}{x}}$ is concave on $(0,1)$, then for arbitrary $x_{i} \in(0,1), i=1,2, \ldots, n$ and from $(30)$, we have

$$
\frac{f\left(x_{1}\right)+f\left(x_{2}\right)+\cdots+f\left(x_{n}\right)}{n} \leq f\left(\frac{x_{1}+x_{2}+\cdots+x_{n}}{n}\right)=f\left(\frac{1}{n}\right)=\left(1-\frac{1}{n}\right)^{n}
$$

and the equality holds if and only if $x_{1}=x_{2}=\cdots=x_{n}=\frac{1}{n}$.

If we rewrite $(31)$ in the form

$$
\left(1-x_{1}\right)^{\frac{1}{x_{1}}}+\left(1-x_{2}\right)^{\frac{1}{x_{2}}}+\cdots+\left(1-x_{n}\right)^{\frac{1}{x_{n}}} \leq n\left(1-\frac{1}{n}\right)^{n},
$$

then, for $n=2$, putting $x_{1}=\nu, x_{2}=\mu$, we obtain exactly (4). So, (32) is a generalization of (4) for arbitrary $n \geq 2$.

Since the sequence $\left\{\left(1-\frac{1}{n}\right)^{n}\right\}_{n=1}^{\infty}$ is strictly increasing and $\lim _{n \rightarrow+\infty}\left(1-\frac{1}{n}\right)^{n}=\frac{1}{e}$, then as a corollary of Theorem 3, we obtain the inequality

$$
\frac{\left(1-x_{1}\right)^{\frac{1}{x_{1}}}+\left(1-x_{2}\right)^{\frac{1}{x_{2}}}+\cdots+\left(1-x_{n}\right)^{\frac{1}{x_{n}}}}{n}<\frac{1}{e} .
$$


But now, we observe that if $x_{i} \in(0,1)$, the (33) holds, i.e., we do not need the condition $x_{1}+x_{2}+\cdots+x_{n}=1$.

Indeed, since $f(x)=(1-x)^{\frac{1}{x}}$ is strictly decreasing on $(0,1)$ (see the Lemma), then

$$
\left(1-x_{i}\right)^{\frac{1}{x_{i}}}<\lim _{x \rightarrow 0^{+}} f(x)=\frac{1}{e} .
$$

Therefore, we have

$$
\frac{\left(1-x_{1}\right)^{\frac{1}{x_{1}}}+\left(1-x_{2}\right)^{\frac{1}{x_{2}}}+\cdots+\left(1-x_{n}\right)^{\frac{1}{x_{n}}}}{n}<\frac{\frac{1}{e}+\frac{1}{e}+\cdots+\frac{1}{e}}{n}=\frac{1}{e}
$$

and (33) is proved.

Finally, we must mention that if $A$ is a fixed IFS over a universe $E$, then we can construct the following two new sets

$$
B=\left\{\left\langle x, \mu_{A}(x)^{\frac{1}{\nu_{A}^{(x)}}}, \nu_{A}(x)^{\frac{1}{\mu_{A}(x)}}\right\rangle \mid x \in E\right\}
$$

and

$$
C=\left\{\left\langle x, \mu_{A}(x)^{\frac{1}{\nu_{A}(x)}}+\nu_{A}(x)^{\frac{1}{\mu_{A}(x)}}, \pi_{A}(x)^{\frac{1}{\pi_{A}(x)}}\right\rangle \mid x \in E\right\} .
$$

These sets are IFSs, because for each $x \in E: \mu_{A}(x)^{\frac{1}{\nu_{A}(x)}}, \nu_{A}(x)^{\frac{1}{\mu_{A}(x)}} \in[0,1]$ and

$$
\mu_{A}(x)^{\frac{1}{\nu_{A}^{(x)}}}+\nu_{A}(x)^{\frac{1}{\mu_{A}(x)}} \leq \mu_{A}(x)+\nu_{A}(x) \leq 1 ;
$$

and $\mu_{A}(x)^{\frac{1}{\nu_{A}(x)}}+\nu_{A}(x)^{\frac{1}{\mu_{A}(x)}}, \pi_{A}(x)^{\frac{1}{\pi_{A}(x)}} \in[0,1]$ and

$$
\mu_{A}(x)^{\frac{1}{\nu_{A}(x)}}+\nu_{A}(x)^{\frac{1}{\mu_{A}(x)}}+\pi_{A}(x)^{\frac{1}{\pi_{A}(x)}} \leq \mu_{A}(x)+\nu_{A}(x)+\pi_{A}(x)=1 .
$$

\section{Conclusion}

In the second part of the present paper, we will represent a new inequality which one may deduce with the help of (4) and the well-known Young's inequality for product. This new inequality also allows IFS interpretation.

\section{References}

[1] Atanassov, K. (1999). Intuitionistic Fuzzy Sets: Theory and Applications. Springer, Heidelberg.

[2] Atanassov, K. (2012). On Intuitionistic Fuzzy Sets Theory. Springer, Berlin.

[3] Fikhtengolts, G. (1965). The Fundamentals of Mathematical Analysis. Vol. 2, Elsevier.

[4] Zadeh, L. (1965). Fuzzy sets. Information and Control, 8(3), 338-353. 